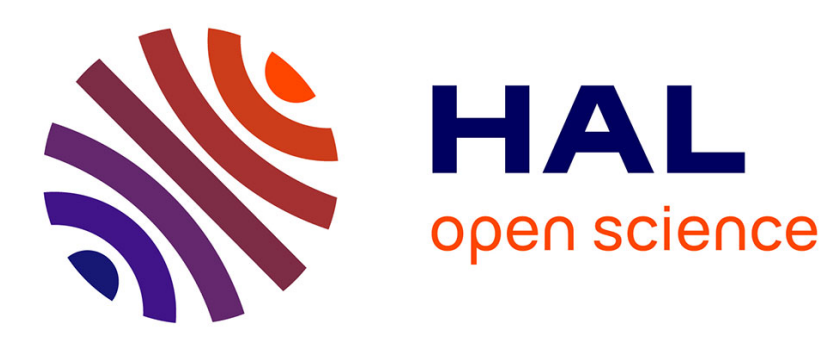

\title{
Color calibration of an RGB camera mounted in front of a microscope with strong color distortion
}

Charrière Renée, Mathieu Hébert, Alain Treméau, Nathalie Destouches

\section{To cite this version:}

Charrière Renée, Mathieu Hébert, Alain Treméau, Nathalie Destouches. Color calibration of an RGB camera mounted in front of a microscope with strong color distortion. Applied optics, 2013, 52 (21), pp.5262-5271. hal-00845866

\section{HAL Id: hal-00845866 https://hal.science/hal-00845866}

Submitted on 18 Jul 2013

HAL is a multi-disciplinary open access archive for the deposit and dissemination of scientific research documents, whether they are published or not. The documents may come from teaching and research institutions in France or abroad, or from public or private research centers.
L'archive ouverte pluridisciplinaire HAL, est destinée au dépôt et à la diffusion de documents scientifiques de niveau recherche, publiés ou non, émanant des établissements d'enseignement et de recherche français ou étrangers, des laboratoires publics ou privés. 


\title{
Color calibration of an RGB camera mounted in front of a microscope with strong color distortion
}

\author{
Renée Charrière, * Mathieu Hébert, Alain Trémeau, and Nathalie Destouches \\ Université de Lyon, Université Jean Monnet de Saint Etienne, CNRS UMR 5516, \\ Laboratoire Hubert Curien, F-42000 Saint Etienne, France \\ *Corresponding author: renee.charriere@gmail.com
}

Received 8 April 2013; revised 12 June 2013; accepted 24 June 2013; posted 25 June 2013 (Doc. ID 188405); published 18 July 2013

\begin{abstract}
This paper aims at showing that performing color calibration of an RGB camera can be achieved even in the case where the optical system before the camera introduces strong color distortion. In the present case, the optical system is a microscope containing a halogen lamp, with a nonuniform irradiance on the viewed surface. The calibration method proposed in this work is based on an existing method, but it is preceded by a three-step preprocessing of the RGB images aiming at extracting relevant color information from the strongly distorted images, taking especially into account the nonuniform irradiance map and the perturbing texture due to the surface topology of the standard color calibration charts when observed at micrometric scale. The proposed color calibration process consists first in computing the average color of the color-chart patches viewed under the microscope; then computing white balance, gamma correction, and saturation enhancement; and finally applying a third-order polynomial regression color calibration transform. Despite the nonusual conditions for color calibration, fairly good performance is achieved from a 48 patch Lambertian color chart, since an average CIE-94 color difference on the color-chart colors lower than 2.5 units is obtained. (C) 2013 Optical Society of America

OCIS codes: (330.1710) Color, measurement; (330.1730) Colorimetry.

http://dx.doi.org/10.1364/AO.52.005262
\end{abstract}

\section{Introduction}

Nowadays, image acquisition devices are very common and make it easy to take a picture to save some scene. But all of us are concerned by the fact that two different cameras, or even the same camera with different settings, give different colors for the same scene, which may even be different from those directly perceived by our visual system. These differences are explained by the fact that the responses of the three RGB sensors of a camera are different from the responses of the retina cells and subsequent analysis in the brain [1,2]. Responses of the sensors also vary from one camera to another. The RGB values given by any imaging device are thus device dependent.

$1559-128 X / 13 / 215262-10 \$ 15.00 / 0$

(C) 2013 Optical Society of America
The color characterization of an imaging device consists in computing the relationship between the RGB values given by the device and color components, which depend only on the reflectance properties of the observed objects and the illumination, in a device-independent color space, such as CIEXYZ or CIELAB [ $\underline{3}, \underline{4}]$. This relationship is generally calculated by acquiring with the considered device a reference color chart with well-known color values in standard illumination conditions [5]. Various types of relationships have been already implemented in the literature, such as multidimensional lookup tables with interpolation [6-8], least-squares polynomial regressions with various polynomial orders, advanced regression modeling [9], neural networks [10,11] and also human-observation-based models [12]. These color calibration methods have been implemented successfully for various imaging systems: computer vision systems for meat quality evaluation $[\underline{9}, \underline{13}]$, 
dermatoscopic imaging systems [14-16], colposcopes [17], commercial digital cameras for various applications such as dentistry [18] or color advice for home décor [19], displays [7,12], or printers $[\underline{8}, \underline{11}]$.

Generally color calibration algorithms are not very robust to changes in the illumination conditions, since the color of objects depends on the illumination source. In our microscope imaging system, the illumination source intensity is not spatially uniform and is very far from standard daylight illuminants, such as D65 or D50 [4]. Moreover, the microscope studied here is dedicated to laser inscription on photosensitive samples. Thus, many optical elements are present on the light path from the illumination source to the observation camera, which may modify the light spectrum and distort the observed colors. An additional problem encountered in microscope color calibration is the nonuniformity of commonly used color charts at micrometric scale. Because of all these particularities of the microscope imaging system, the previously cited color calibration methods cannot be directly applied on the colors extracted from the microscope camera images. A prior preprocessing of the camera images is needed, first to extract the average color of the nonuniform images of the color-chart patches under the microscope and then to compute white balance, gamma correction, and saturation enhancement of these extracted colors. The color calibration algorithm applied after precorrection is a third-order polynomial regression which converts camera colors, previously converted into the CIELAB color space, with the hypothesis that the camera RGB color components are sRGB [2] color components, to CIELAB device-independent color components. It has been demonstrated [20] that polynomial transformations and neural networks give similar performance, but polynomial transformations are easier to implement. And, as with neural networks, three-dimensional (3D) lookup tables require a large number of reference samples to achieve good performances. That is why we will use a polynomial color calibration transform.

After color calibration with a color chart composed of a selection of 48 pieces of Munsell Matte Color Sheets, the average CIE-94 color difference [21] on the color-chart patches between the corrected colors extracted from the microscope camera images and the reference colors of the color chart is lower than 2.5 units. The reference colors of the color chart are computed by taking into account the optical configuration of the microscope and its particular illuminant. To compare with the literature, the color difference may be expressed in term of CIE-76 color difference [22]. The obtained average CIE-76 color difference is lower than 4.4 units, which is below the accepted tolerance in the printing industry, which is 6 units [23], and for television cameras, which is 5 units [24]. This result is worse than those obtained for example in $[9,13]$, but in these references the color patches were taken directly with a camera, without the specific problems induced by the microscope. In the field of dermatoscopic imaging, where the images are taken under an epiluminescence microscope, color calibration gives CIE-76 color differences ranging from 2.5 to 3.9 units, depending on the camera used to acquire the images [16]. It is nevertheless to be noticed that in [16], contrary to $[9,13]$, the color difference is computed on color patches different from those used for the calibration. In [25], a microscope imaging system was calibrated, including the calibration of the screen where the images were displayed. As we were interested only in the RGB values given by the camera, the study of the proper restitution of the colors by the computer screen to which the camera is connected is not beyond the scope of this article, but it is interesting to compare our color calibration quality with that obtained in [25]. The color difference, expressed in term of CIEDE2000 color distance [26] between displayed and reference colors, is better than 4.8. In terms of CIEDE2000 color distance, our average color difference is lower than 2.5 units. In the following, the color difference will always be expressed in terms of CIE-94 color difference because it provides a better agreement with the human perceived color difference [2] than the CIE-76 formula and is more commonly used in this domain than the CIEDE2000 color distance.

In the following, we first describe the optical scheme of the microscope. Then, we detail the color calibration method: the choice of the ground truth colors used for the color calibration, the color extraction from the microscope camera images, and the color calibration transformation. Next, the influence of the chosen illuminant on the calibration performance will be studied, and finally the robustness of our color calibration method will be discussed.

\section{Optical Description of the Microscope}

The microscope used in this study is an Olympus BX51M, which has been modified to perform laser inscriptions and spectroscopic measurements on photosensitive samples. The latter exhibit various colors, depending on the laser exposure conditions [27], which can be characterized by spectroscopic measurements. Nevertheless, measuring the colors directly with a calibrated camera placed in front of the microscope significantly saves time. That is why a color calibration of the system composed of the microscope and the observation camera has been implemented.

The scheme of the microscope is presented on Fig. 1. The samples are observed with a 8 bit camera (ref. IDS UI-2240-C) connected via a USB cable to a computer, through a 10x objective lens (MPLN 10x from Olympus). The eyepiece is an Olympus U-TV1x-2 with $1 \times$ magnification. The sample is illuminated by a halogen lamp with an optical power of $100 \mathrm{~W}$.

The microscope has three more optical entries: a first one brings the laser beam, which changes the color of photosensitive samples; a second one brings a broadband laser-driven light source (ref. Energetiq EQ-99FC), which illuminates the sample during 


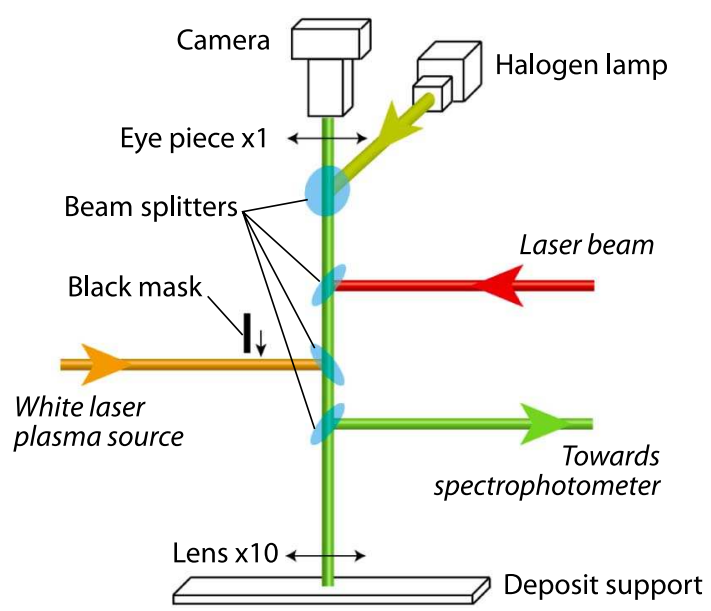

Fig. 1. Optical scheme of the microscope used for laser inscription on coated glass plates and spectroscopic measurements of the inscribed patterns.

spectroscopic measurements; and a third one extracts the light reflected by the sample toward the input of a spectrometer. Unfortunately, the halogen lamp cannot be used to make spectroscopic measurements because its signal is too low in the short wavelength domain of the visible spectrum. The 50-50 beam splitters corresponding to these three optical entries are Melles Griot BTF-VIS-50-5001 M-C for the laser beam and Thorlabs BSW27 for the laser plasma light source and the spectrometer.

To prevent damage of the camera due to too strong fluxes, the samples are not observed during laser inscription. The laser is blocked by a shutter placed on the path of the beam before the microscope (not represented on Fig. 1). The only source of illumination of samples during observation through the camera is the halogen lamp, the white laser plasma source being used only during spectroscopic measurements.

As several optical entries are necessary for our experiment, the illumination light, as the reflected light from the sample, crosses many optical elements that modify its spectrum. Indeed, manufacturer specifications indicate transmittance variations over the visible spectrum of about $20 \%, 10 \%$, and $4 \%$, respectively, for the 10x objective lens, the Thorlabs beam splitter, and the Melles Griot beam splitter. The color calibration has then to take into account the influence of all these optical elements, but, as their characteristics are not well known, their influence will be considered globally. This is simply done by taking images with the camera of patches of calibrated colors put in place of the samples in the microscope. The residual chromatic aberration of the objective lens may also induce a variation of the colors of the camera images with the focusing. The focus is adjusted by optimizing the sharpness of the camera images. Each time the color chart is moved, for example switching to the next color patch, the focus is reoptimized. It has been verified, for 10 acquisitions of the same color patch, that the repeatability uncertainty of the focusing induces negligible color variations: the corresponding CIE-94 color difference is below 0.5 .

Note that, as the spectral responses of the R, G, B sensors of the camera cannot be precisely measured, the color calibration cannot be made in the spectral domain.

\section{Choice of the Color Chart for Calibration}

The colorimetric calibration of the system \{camera + optical system \} needs to establish the correspondence between RGB device-dependent color values given by the camera and color values depending only on the spectral reflectance of the observed object and the spectral irradiance. This transformation can be determined by capturing images of some color patches with well-known spectral reflectance, and thereby well-known color values.

The choice of an appropriate color chart is crucial. It must be adapted both to the spectrophotometer for spectral measurements and to the imaging system, and its colors must correctly sample the color space. The spectrophotometer generally captures light from a large surface, for instance a disk of 5 to $10 \mathrm{~mm}$ diameter, and integrates all surface heterogeneities. The measuring geometry may have a strong influence if the surface of the color chart is glossy. For comparison, we used three different geometries: the $45^{\circ}: 0^{\circ}$ geometry (X-Rite i1 Pro spectrophotometer) where light comes at $45^{\circ}$ and reflected light is captured in the direction normal to the surface; the de: $8^{\circ}$ geometry where the incident light is diffuse and the reflected light is captured at $8^{\circ}$ from the normal to the surface where no light can be specularly reflected by the surface to the detector, and the de: $8^{\circ}$ geometry where the incident light is diffuse and the reflected light is captured at $8^{\circ}$ from the normal to the surface where light can be specularly reflected by the surface to the detector [28]. The $45^{\circ}: 0^{\circ}$ and de: $8^{\circ}$ geometries do not capture specularly reflected light, in contrast with the de: $8^{\circ}$ geometry and the $0^{\circ}: 0^{\circ}$ geometry on which our microscope is based. The de: $8^{\circ}$ geometry is therefore preferable to get comparable information from our spectral measurements and our captured images.

The color chart must also be adapted to the imaging system. In the case of microscope imaging, the color patches should be as smooth as possible at a micrometric scale. This discards charts with glossy, grainy surface such as the X-Rite Digital Color Checker SG chart, which displays too contrasted texture when viewed under microscope. When the imaging system, as in our case, is based on the $0^{\circ}: 0^{\circ}$ geometry, patches with a flat surface will reflect two light components: uncolored light specularly reflected by the surface, and colored light issued from the patch's bulk. Matte samples are preferable since the uncolored light reflected by the surface is diffused and a smaller amount of it is therefore captured by the camera at $0^{\circ}$. We thus chose a selection of 48 pieces of Munsell Matte Color Sheets as presented in Fig. 2, almost Lambertian and with 


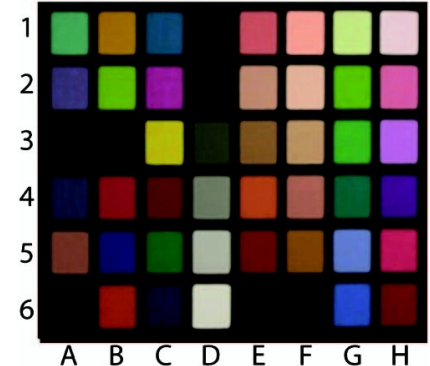

Fig. 2. Picture of the 48-patch color chart based on Munsell Matte Color Sheets.

low surface texture, composed of 24 colors close to the colors of the standard X-Rite ColorChecker and 24 additional colors to increase the sampling of the color space.

\section{Ground Truth Colors of a Chart Obtained from Spectral Measurement}

The first step of the calibration process is the determination by spectral measurements of the color values of the patches of the color chart. A lot of color spaces can be chosen to express these components, but it is judicious to select the color space in which the color values delivered by the camera are expressed. In our case, the camera delivers values in the sRGB space. The conversion of spectra into sRGB values needs to define the illuminant, which is by default the D65 illuminant in the sRGB color space. In this microscope, the light illuminating the sample comes from a halogen lamp and crosses the whole optical system. Its exact spectral power distribution (SPD) cannot be measured since it would need to place the input of a spectrophotometer in place of the sample, which is not possible. We will first assume that the closest illuminant to the incident light is the A illuminant [4]. The question of the illuminant is further addressed in Section $\underline{8}$. Let us explain how spectral measurements are converted into sRGB values with A illuminant.

The sRGB color components are calculated in the following way. First, the reflectance spectra measured with the spectrophotometer are converted into $X Y Z$ values, as defined by the CIE [29]:

$$
\left\{\begin{array}{l}
X=k \sum_{i=1}^{n} \bar{x}\left(\lambda_{i}\right) R\left(\lambda_{i}\right) S_{A}\left(\lambda_{i}\right) \\
Y=k \sum_{i=1}^{n} \bar{y}\left(\lambda_{i}\right) R\left(\lambda_{i}\right) S_{A}\left(\lambda_{i}\right), \\
Z=k \sum_{i=1}^{n} \bar{z}\left(\lambda_{i}\right) R\left(\lambda_{i}\right) S_{A}\left(\lambda_{i}\right)
\end{array}\right.
$$

where $\lambda_{i}$ denotes the different wavelengths labeled from 1 to $n ; \bar{x}(\lambda), \bar{y}(\lambda)$, and $\bar{z}(\lambda)$ are standard observer color matching functions defined by the CIE; $R(\lambda)$ is the measured spectral reflectance of the sample, $S_{A}(\lambda)$ is the SPD of the $A$ illuminant, and

$$
k=\frac{100}{\sum_{i=1}^{n} \bar{y}\left(\lambda_{i}\right) S_{A}\left(\lambda_{i}\right)} .
$$

The $X Y Z$ values are then converted into sRGB values according to the nonlinear transform, denoted
$T_{X Y Z \rightarrow \text { sRGB }}$, which may be found for example in [30]. Since the basic illuminant for sRGB is the $\overline{\mathrm{D} 6} 5$ illuminant, a so-called Bradford matrix transform must be applied before the transform $T_{X Y Z \rightarrow \text { sRGB }}$, converting the $X Y Z$ values defined with the $A$ illuminant into values corresponding to a D65 illuminant. The Bradford matrix is:

$$
\mathbf{M}_{B}=\left[\begin{array}{ccc}
0.8447 & -0.1179 & 0.3949 \\
-0.1366 & 1.1041 & 0.1292 \\
0.0799 & -0.1349 & 3.1926
\end{array}\right]
$$

The sRGB color values are then obtained with the following relation:

$$
\left[\begin{array}{c}
R \\
G \\
B
\end{array}\right]=T_{X Y Z \rightarrow \mathrm{sRGB}}\left(\mathbf{M}_{B} \cdot\left[\begin{array}{c}
X \\
Y \\
Z
\end{array}\right]\right) .
$$

Depending on the measuring geometry of the spectrophotometer used for the measurement of $R(\lambda)$, different RGB values can be obtained. However, with a matte chart such as the one selected in this work, the differences are low as shown with the average and maximal differences for the 48 patches of the color chart, expressed in terms of CIE-94 color distance (hereinafter denoted as $\Delta E_{94}$ ), presented in Table 1.

In the following, we will only use the spectral measurements based on the di: $8^{\circ}$ geometry, carried out with an X-rite Color i7 spectrophotometer in specular component included mode. The RGB values deduced from them will be considered as the ground truth color values of the color chart.

\section{Color Values of the Chart Deduced from the Camera}

The patches of the color chart are placed in front of the microscope and images are saved. The microscope observes only an area of about $590 \times 460$ micrometers. Even though the patch seems uniform at first sight, a texture appears at macroscopic scale, illustrated in Fig. 3(a) through the example of the cyan patch in the top left corner of the color chart (see Fig. 2). The size of the images is $1280 \times 1024$ pixels. The results presented below are for images acquired in jpeg format, but no significant variations of the color correction accuracy have been observed by saving the images in bitmap or Bayer pattern encoded formats, the two other formats allowed by the camera.

Table 1. Difference between Colors Issued from Spectral Measurements Based on Different Geometries

\begin{tabular}{lcc}
\hline Compared Geometries & Average $\Delta E_{94}$ & Maximal $\Delta E_{94}$ \\
\hline $45^{\circ}: 0^{\circ}$ and de $: 8^{\circ}$ & 0.66 & 1.54 \\
$45^{\circ}: 0^{\circ}$ and di $: 8^{\circ}$ & 0.65 & 1.09 \\
di $: 8^{\circ}$ and de $: 8^{\circ}$ & 0.37 & 0.53 \\
\hline
\end{tabular}




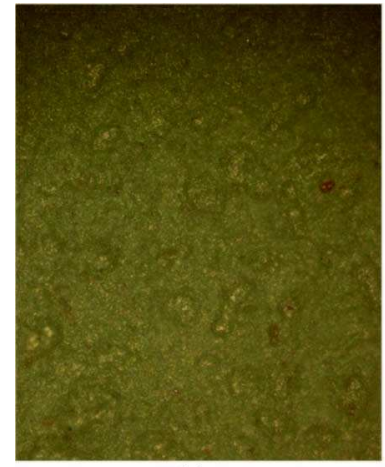

(a)

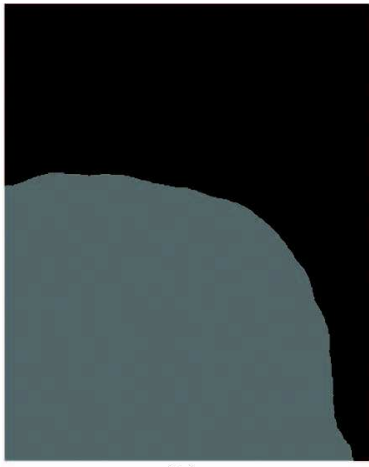

(b)
Fig. 3. (a) A1 cyan patch (see Fig. 2) captured by the camera through the microscope. Here, lightness is magnified by $50 \%$ to highlight surface heterogeneities. (b) Area of interest (gray) in the captured images in which the sample is assumed to be uniformly illuminated.

Every automatic color correction in the camera settings was disabled in order to be sure that capture settings are the same for all patches. The gamma correction was also disabled. The exposure time of the camera as well as the illumination power of the microscope were set to a referenced fixed value. The illumination power of the microscope is approximately set to half of its maximal value, and the exposure time of the camera is chosen by maximizing the signal for the white patch while paying attention that none of the three channels R, G, or B is overexposed. It is also checked that none of the three channels is underexposed for the black patch. Without any automatic color correction, the camera delivers a very brownish image, very far from the color perceived by any observer under a daylight illuminant. Moreover, one can notice that the irradiance is not uniform over the whole viewed surface. A spatial correction of the nonuniformity of the irradiance is indeed difficult to implement in our case, as it would imply finding a sample, spatially uniform under the microscope and having the same irradiance drift law, that is, the same diffusion properties, as the color chart. Further investigations in that domain are not beyond the scope of this article. In order to determine which pixels are more representative of the colors of the patch, we select an area where the irradiance varies as little as possible. This area is determined as follows.

Since the irradiance map on the viewed area is independent of the color chart, we get it on the average image computed from the 48 images of patches of the chart. In order to remove remaining noise due to surface heterogeneities and random artifacts, a mean filtering is performed, based on a $100 \times 100$ pixel convolution kernel. Among the set of RGB values present in the filtered images, the most frequent one is considered as the most representative of the color of the chart as viewed by the camera. Then, the RGB values of each pixel of the image are compared to this more frequent RGB value, and a color distance is expressed in $\Delta E_{94}$. All pixels whose color distance is less than 2 units are aggregated to the area of interest. The area of interest, computed only once, is shown in Fig. 3(b). Figure 10(a) in the Appendix $\underline{A}$ gives the comparison between the ground truth colors of the 48 color chart patches issued from the spectral measurement (in the small triangular insets) and the corresponding colors obtained from the noncalibrated camera.

\section{Preprocessing of the Colors Issued from the Camera}

After color calibration, the camera should provide colors close to those obtained by spectral measurement. The color calibration means determining the transform $U$ between source RGB colors (from the noncalibrated camera) and the target ones (ground truth colors from spectral measurement). Since in our case the source colors are far from the target ones, performing calibration cannot be done using a single transformation function. Instead, a four-step process is proposed, comprising white balance correction, gamma correction, saturation enhancement, and then color transform in itself.

The white balance correction is computed by multiplying each R, G, or B value of a source color by the respective ratios $R_{D 6}^{\text {mes }} / R_{D 6}^{\text {cam }}, G_{D 6}^{\text {mes }} / G_{D 6}^{\text {cam }}$, or $B_{D 6}^{\text {mes }} / B_{D 6}^{\text {cam }}$, where $X_{D 6}^{\text {mes }}$ denotes the target $\mathrm{R}$, $\mathrm{G}$, or $B$ value of the white patch D6 of the color chart issued from spectral measurement and $X_{D 6}^{\mathrm{cam}}$ denotes source $\mathrm{R}, \mathrm{G}$, or $\mathrm{B}$ value of the same patch issued from the camera.

The gamma correction and the saturation enhancement are performed as follows. The camera RGB values after white balance correction and the ground truth colors are converted into color values in the CIELAB color space by assuming that the RGB camera colors are in the sRGB color space. Then, for each patch $j$, the lightness component $L_{j}^{\text {mes }}$ of the ground truth color is plotted versus the lightness component $L_{j}^{\text {cam }}$ of the white balance corrected camera color. The 48 obtained points are then fitted with a second-order polynomial function as

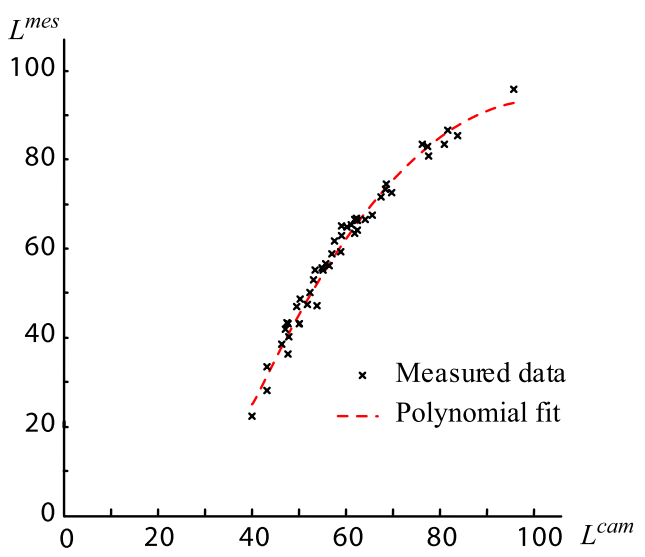

Fig. 4. Gamma correction of lightness after correction of the white balance. The fit curve is given by the polynomial equation $L^{\mathrm{mes}}=-0.0184\left(L^{\mathrm{cam}}\right)^{2}+3.717 L^{\mathrm{cam}}-94.616$. 


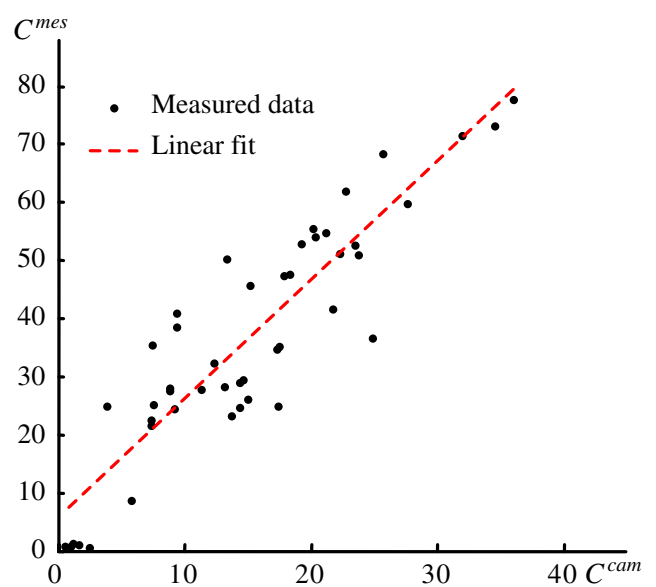

Fig. 5. Saturation enhancement of chroma after correction of the white balance. The fit curve is given by the equation $C^{\text {mes }}=2.052 C^{\text {cam }}+5.665$.

shown in Fig. 4. By applying this polynomial function to the $L$ components of the camera colors, the lightness of every color will be closer to the lightness of the corresponding ground truth color obtained by spectral measurement.

The chroma component $C$ of a color expressed in the CIELAB color space is defined by the following equation:

$$
C=\sqrt{a^{2}+b^{2}}
$$

For each patch $j$, the chroma component $C_{j}^{\text {mes }}$ of the ground truth color is plotted versus the chroma component $C_{j}^{\mathrm{cam}}$ of the white balance corrected camera color. The 48 obtained points are represented in Fig. 5. One can notice that the linear correlation between these two variables is quite strong, with a Pearson's correlation coefficient of 0.9 . This would have been different if we had plotted the $C_{j}^{\text {mes }}$ values versus the chroma components of the camera colors before the white balance correction. The $C_{j}^{\text {cam }}$ values are lower than the $C_{j}^{\text {mes }}$ values by a factor of 2 . Thus, in order to enhance the saturation of the camera colors, the variations of $C_{j}^{\text {mes }}$ versus $C_{j}^{\text {cam }}$ are fitted by a straight line, and the equation of this straight line is applied to the chroma components of all colors captured by the camera.

Figure 6 recaps the color corrections performed in this preprocessing of the camera images. The gamma correction and saturation enhancement equations are deduced from the fits presented in Figs. $\underline{4}$ and 5. Figure 10 (in the Appendix A) shows a comparison between the ground truth colors of the 48 color chart patches and, in Fig. 10(b), the camera colors after white balance correction and in Fig. 10(c), the camera colors after gamma correction and saturation enhancement. The average color distance between these latter and the ground truth colors is $\Delta E_{94}=5.56$ units, that is, about twice lower than the average distance computed before correction, which was 11.62 units; the maximal distance is $\Delta E_{94}=14.14$ units (for the green patch G4).

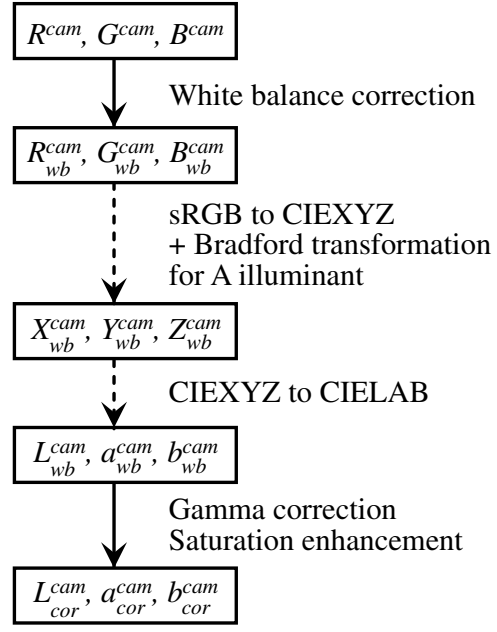

Fig. 6. Color precorrection process applied to a camera image. Dashed arrows indicate changes of color space and solid arrows indicate color precorrections.

We can now calculate the color transform $U$ from the corrected camera colors to the ground truth ones.

\section{Color Transform}

For patch $j$, we denote as $\mathbf{v}_{j}^{\text {mes }}=\left(L_{j}^{\text {mes }}, a_{j}^{\text {mes }}, b_{j}^{\text {mes }}\right)$ the color vector expressed in the CIELAB color space representing the ground truth color issued from spectral measurements and as $\mathbf{v}_{j}=\left(L_{j}, a_{j}, b_{j}\right)$ the vector representing the color captured by the camera after white balance, gamma, and saturation corrections. We are searching for a transform $U$ converting the vectors $\mathbf{v}_{j}$ into vectors as close as possible to the vectors $\mathbf{v}_{j}^{\text {mes }}$. Many solutions may be possibly obtained for $U$ because of the large number of varying parameters in the optimization process. It will be shown below that the obtained different transforms lead to similar color calibration accuracies.

The transformation $U$ should compensate all color distortions due to the capture device and should therefore provide colors depending only on the properties of the observed object and the illuminant. Since we have a set of only 48 colors in the whole color space, we must pay attention to possible divergence of the color calibration transformation between the colors of the patches. The selected transform is a polynomial transform, as neural networks or 3D lookup tables require a large number of reference samples to work properly. The higher the order of the polynomial, the better will be the correction of the colors of the patches, but the higher will be the risk of divergence between these colors. A good compromise seems to be a third-order polynomial [20]. $U$ is therefore a thirdorder polynomial function of the three variables $L_{j}^{c c}$, $a_{j}^{c c}$, and $b_{j}^{c c}$. It can then be written as the following matrix operation:

$$
\mathbf{v}_{j}^{\text {estim }}=\mathbf{U} \cdot \mathbf{w}_{j}^{T},
$$

where $\mathbf{U}$ is a $3 \times 20$ matrix defined as 


$$
\mathbf{U}=\left[\begin{array}{llll}
u_{1}^{L} & u_{2}^{L} & \cdots & u_{20}^{L} \\
u_{1}^{a} & u_{2}^{a} & \cdots & u_{20}^{a} \\
u_{1}^{b} & u_{2}^{b} & \cdots & u_{20}^{a}
\end{array}\right]
$$

$\mathbf{w}_{j}$ is a vector of size 20 defined from $L_{j}, a_{j}$, and $b_{j}$ values issued from the camera image after the three preprocessing corrections:

$$
\begin{gathered}
\mathbf{w}_{j}=\left(1, L_{j}, a_{j}, b_{j}, L_{j}^{2}, L_{j} a_{j}, L_{j} b_{j}, a_{j}^{2}, a_{j} b_{j}, b_{j}^{2}, L_{j}^{3}, L_{j}^{2} a_{j},\right. \\
\left.L_{j}^{2} b_{j}, L_{j} a_{j}^{2}, L_{j} a_{j} b_{j}, L_{j} b_{j}^{2}, a_{j}^{3}, a_{j}^{2} b_{j}, a_{j} b_{j}^{2}, b_{j}^{3}\right),
\end{gathered}
$$

the symbol $T$ denotes the matrix transpose, and $\mathbf{v}_{j}^{\text {estim }}=\left(L_{j}^{\text {estim }}, a_{j}^{\text {estim }}, b_{j}^{\text {estim }}\right)$ represents in the CIELAB space the color of patch $j$ estimated through the transform $U$.

The entries of matrix $\mathbf{U}$ are determined by minimizing the following sum:

$$
\sigma=\sum_{j=1}^{m} \Delta E_{94}\left(\mathbf{v}_{j}^{\mathrm{mes}}, \mathbf{v}_{j}^{\mathrm{estim}}\right)+\sum_{s \in\{L, a, b\}} \sum_{k=1}^{20}\left|u_{k}^{s}(o(k)) !\right|,
$$

where $o(k)$ denotes the total power of the $k$ th term of the polynomial in $L_{j}, a_{j}$, and $b_{j}$ [e.g., $o(k)=2$ for the term $L_{j}^{2}$ and $o(k)=3$ for the term $\left.L_{j}^{2} a_{j}\right], n$ ! is the factorial of integer $n, \Delta E_{94}\left(\mathbf{v}_{j}^{\text {mes }}, \mathbf{v}_{j}^{\text {estim }}\right)$ is the CIE-94 color distance between the colors represented by the vectors $\mathbf{v}_{j}^{\text {mes }}$ and $\mathbf{v}_{j}^{\text {estim }}$, and $m$ is the number of color patches in the chart, 48 in our case. The second term of the sum $\sigma$ is added to constrain the optimization with low values for the polynomial coefficients corresponding to terms with high total power. The purpose of doing so is to avoid divergences of the color calibration transformation for colors that are not present in the color chart. A widely used method to solve the coefficients of a polynomial transformation is the least-square regression. But this method corresponds to a minimization of the Euclidian distance in the color space where it is applied, whereas the minimization of $\sigma$ leads to a direct minimization of the average CIE-94 color distance, which better corresponds to visual perception.

The minimization of $\sigma$ is numerically computed with the fminsearch function of Matlab, which uses the Nelder-Mead simplex algorithm [31]. In comparison to the least-square regression, we observed that our method, although slower, is more accurate. This algorithm needs a starting point to compute the optimization. In order to obtain the best optimization independently of the starting point, we proceed as follows by computing many iterations of the optimization: for the first iteration, the starting point $U_{0}$ is obtained by calculating the transform $U^{\prime}$ for a second-order polynomial in $L_{j}, a_{j}$, and $b_{j}$. $U^{\prime}$ is obtained by solving, in a least-square sense, the equation

$$
\left[\begin{array}{cccc}
L_{1}^{\text {mes }} & L_{2}^{\text {mes }} & \cdots & L_{m}^{\text {mes }} \\
a_{1}^{\text {mes }} & a_{2}^{\text {mes }} & \cdots & a_{m}^{\text {mes }} \\
b_{1}^{\text {mes }} & b_{2}^{\text {mes }} & \cdots & b_{m}^{\text {mes }}
\end{array}\right]=\mathbf{U}^{\prime} \cdot\left[\begin{array}{c}
\mathbf{w}_{1}^{\prime} \\
\mathbf{w}_{2}^{\prime} \\
\vdots \\
\mathbf{w}_{m}^{\prime}
\end{array}\right]^{T},
$$

where $\mathbf{w}_{j}^{\prime}$ is a vector of size 10 containing the terms of a second-order polynomial in $L_{j}, a_{j}$, and $b_{j}$ :

$$
\mathbf{w}_{j}^{\prime}=\left(1, L_{j}, a_{j}, b_{j}, L_{j}^{2}, L_{j} a_{j}, L_{j} b_{j}, a_{j}^{2}, a_{j} b_{j}, b_{j}^{2}\right) .
$$

The terms of $U_{0}$ whose order is lower than or equal to 2 are set equal to the values of $U^{\prime}$. The third order terms of $U_{0}$ are set to 0 . For this first iteration, the best transform $U$ is computed by minimizing the value of $\sigma$, as described above.

For the following iterations, the starting point is obtained by modifying the transformation obtained by the previous iteration. $\tilde{U}$ denotes the best transformation computed from the previous iteration, $\left\{\tilde{u}_{k}^{s}\right\}_{s \in\{L, a, b\}, k \in\{1 \ldots 20\}}$, its matrix entries, $U_{0}$; the starting point transformation for the current iteration; and $\left\{u_{0, k}^{s}\right\}_{s \in\{L, a, b\}, k \in\{1 \ldots 20\}}$, its matrix entries. $U_{0}$ is computed with the following operation:

$$
u_{0, k}^{s}=\tilde{u}_{k}^{s}+\kappa \varepsilon_{k}^{s} \tilde{u}_{k}^{s},
$$

where $\varepsilon_{k}^{s}$ is a random number with a probability density function constant over $[-1,1]$, and $\kappa$ is a constant whose value has been experimentally optimized to produce the best results for minimization of $\sigma$.

Once the new starting point $U_{0}$ is calculated, the minimization of $\sigma$ is performed with the fminsearch function. If the result for $\sigma$ is better than the result obtained with the previous iteration, the new best transformation is kept and is used for the computation of the starting point for the next iteration. Otherwise, the new best transformation is thrown and the starting point for the next iteration is calculated from the previous best transformation, but with a new drawing of the random values $\varepsilon_{k}^{s}$.

The total number of iterations, as well as the value of the constant $\kappa$, were manually selected. If $\kappa$ is too high, the starting points of the optimization are far from each other, and also far from the optimal starting point. Only a few iterations give good results, so the final value of $\sigma$ will not really be better than the value obtained with the first iteration unless we drastically increase the number of iterations. If $\kappa$ is too low, the starting points are almost the same after each iteration and $\sigma$ is not minimized. For the results presented in the present work, the value of $\kappa$ was set to 0.1 and the number of iterations was set to 50 .

In order to verify that these values provide a minimal value for $\sigma$, we tested the algorithm 10 times on the 48 patches of the color chart and observed the variations of $\sigma$ after the first iteration (the one where the starting point is obtained with a least-square resolution on a second-order polynomial) and after 
50 iterations (with $\kappa=0.1$ ). After the first iteration, the value of $\sigma$ was 141 in each test. After 50 iterations, fluctuations of the value of $\sigma$ are weak: it was between 119 and 125 with a relative standard deviation, that is a ratio of standard deviation to average, of $1.5 \%$. We can therefore assume that the selected values for $\kappa$ and the number of iterations are relevant for good optimization of the transform $U$.

The average $\Delta E_{94}$ color distance between the 48 ground truth colors and camera colors after precorrections and color calibration indicates the performance of the color calibration: it was 2.32 units in all tests with almost no fluctuations, therefore twice lower than before the $U$ transform, and the maximal distance, observed for the D1 black patch, varies between 10 and 12 units while it was 14.14 units before the $U$ transform. Figure 10(d) (in the Appendix A) shows a comparison between the ground truth colors of the 48 color-chart patches and the camera colors after precorrections and color calibration.

\section{Influence of the Illuminant}

The previous calibration has been computed with an illuminant $A$, which corresponds to the light emitted by a blackbody of temperature $2856 \mathrm{~K}$. But since the exact SPD of the incident light, filtered by the optics and the beam splitters, is not known, there is some interest to see whether other illuminants would yields better calibration performance. We thus tested several illuminants corresponding to blackbody radiations with different temperatures. We have thus compared the prediction accuracy obtained with a blackbody illuminant for various temperature values.

Figure 7 shows the variations of the average $\Delta E_{94}$ color distance between the camera colors after color calibration and the ground truth colors as a function of the blackbody illuminant temperature. Despite a noisy curve due to the fact that random starting points are selected at the beginning of the optimization iterations, a minimum is displayed around $2000 \mathrm{~K}$, which corresponds to a rather reddish illuminant and is consistent with the brownish colors rendered by the camera before correction. In the following, a blackbody illuminant of temperature $1900 \mathrm{~K}$ is selected.

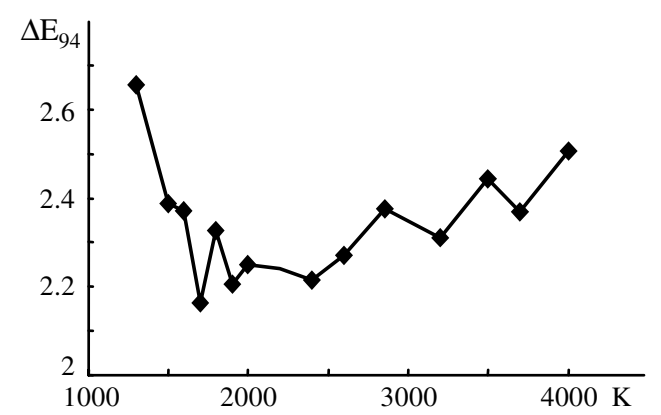

Fig. 7. Variations as a function of the blackbody temperature of the illuminant of the average $\Delta E_{94}$ color distance between ground truth colors and camera colors after preprocessing corrections and color calibration.

\section{Robustness of the Color Calibration}

In the previous section, we presented the best calibration accuracy we could achieve. The average $\Delta E_{94}$ color distance between ground truth colors and corrected camera colors on the 48 patches of the calibration chart is 2.20 units. The maximal distance is 10.98 units for the black patch D1. The bad correction for this patch may be due to its low saturation value, which makes its hue very sensitive to fluctuations, as well as to the fact that this patch is situated at the edge of the volume of the color space defined by the color coordinates of the color-chart patches.

We now propose to compare full images before and after color correction. Five examples are shown in Fig. 8. We see that purple noise appears in the corrected image of the black patch $\mathrm{D} 1$, which is linked to the fact that this patch is the worst corrected. Recall that the color correction method relies on an average color of each patch of the chart, computed from all pixels of the uniformly illuminated area represented in Fig. 3(b). If we now consider an image (e.g., of a patch of the chart), it is equivalent to apply the color correction to the individual pixel colors in the uniformly illuminated area, then average the corrected colors, or to average the individual pixel colors, then apply the color correction. In the absence of texture on the surface or of noise in the image, both methods would yield similar performance, but it may happen that small color textures are emphasized by the color correction (we see for example some purple pixels in the corrected image of the black patch; see Fig. 8). We observe that, in the case of the 48 patches of the calibration chart, better accuracy is achieved with the first method while the second method gives an average color distance of 2.53 units (to compare with 2.20 units with the first method) and a maximal color distance of 8.77 units again for the black patch (to compare with 10.98 units with the first method) for which averaging after the color correction limits the influence of the purplish noise pixels.

We also tested the robustness of our color calibration algorithm on 41 samples of a matte paint color chart: these colors were different from those used to compute the color correction transform (see Fig. $\underline{9}$ ).

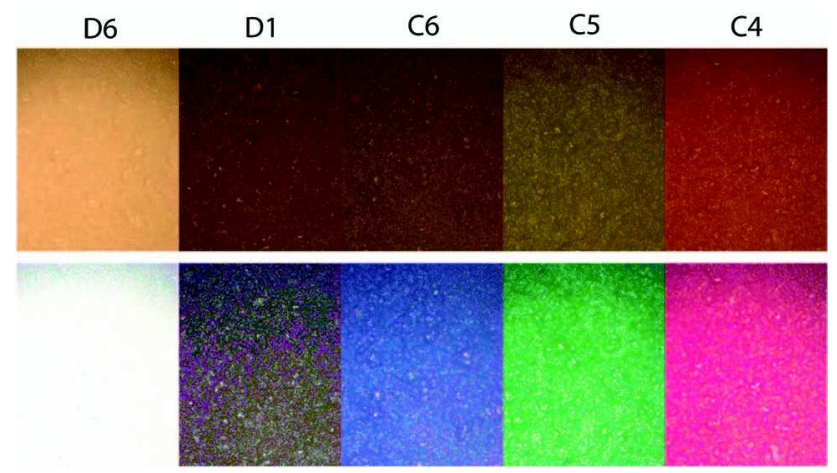

Fig. 8. Images of five Munsell Matte Color Sheets captured by the camera before correction (top) and after corrections (precorrections and color calibration with a blackbody illuminant at $1900 \mathrm{~K}$ ). 


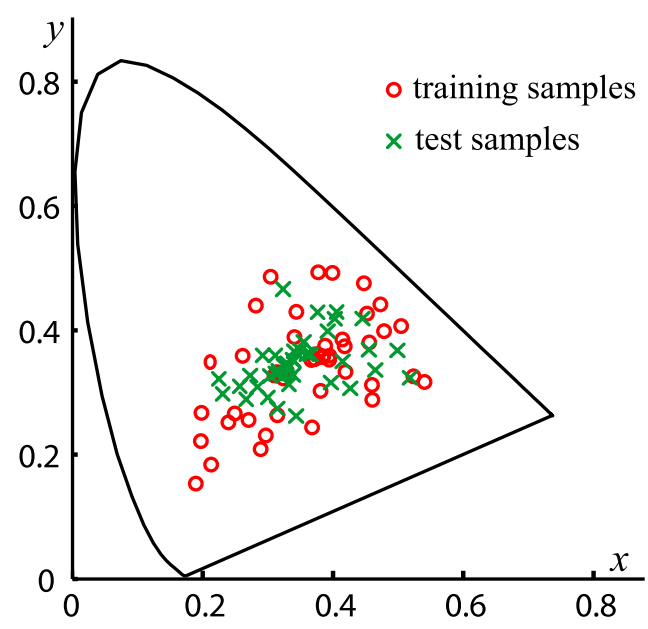

Fig. 9. Representation of the $x-y$ coordinates of the training and test samples in the CIE 1931 chromaticity diagram. For sake of clarity the illuminant chosen for this representation is the standard D65.

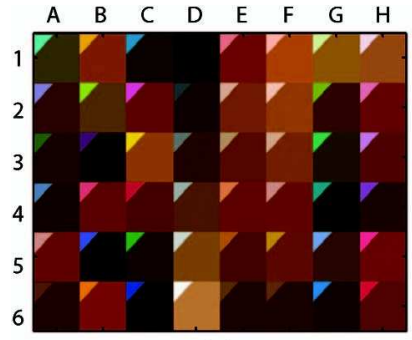

(a)

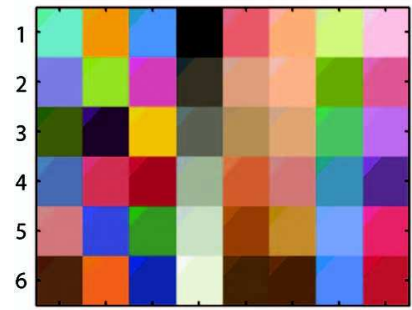

(c)

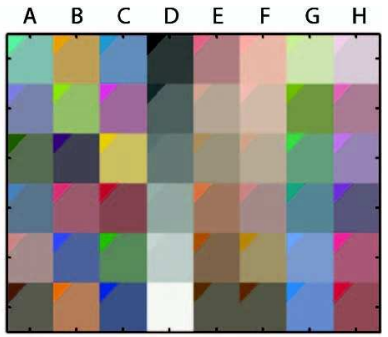

(b)

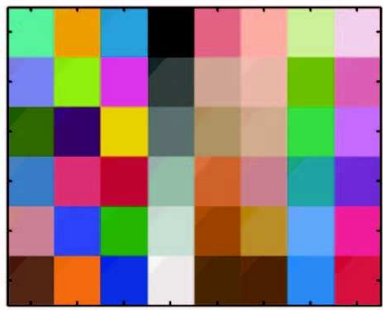

(d)
Fig. 10. Comparison between the ground truth colors, calculated for an illuminant $A$, of the 48 color-chart patches issued from the spectral measurement (in the small triangular insets) and the corresponding colors: (a) obtained from the noncalibrated camera, (b) after white balance correction, (c) after white balance, gamma correction, and saturation enhancement, and (d) after color transform $U$.

These samples were selected for their homogeneity at microscopic scale and their almost Lambertianreflector quality. The average and maximal $\Delta E_{94}$ color distance on the test samples are respectively 3.1 units and 12.2 units, which confirms that our method performs outside the set of colors used for its calibration.

However, the strong color correction presented here increases the image noise. By capturing the same image 10 times, selected so that the average value of each $R, G$, and $B$ channel is approximately equal to $255 / 2$ after color corrections, we observe that the noise level increases from about 1 before corrections to about 5 thereafter. Further improvements of our color correction method should include noise reduction algorithms.

\section{Conclusion}

We have presented a color calibration method for a camera mounted in front of a microscope based on the acquisition of an almost Lambertian 48 patch color chart. This calibration has been performed in very bad conditions: first, the microscope camera used is a low-cost camera, and second, as the microscope is dedicated to laser inscription many optical elements are present on the light path. Moreover the microscope illumination is a simple halogen lamp. So the colors of the patches obtained under the microscope are all very brownish, far from the colors of the patches perceived by a human observer looking directly at the color chart illuminated for example by a fluorescent lamp. Furthermore, the color patches are not smooth and uniform at the microscope scale.

In order to cope with these issues, our calibration method relies on three steps: extraction of the color of the patches from microscope images, precorrections (white balance, gamma, and saturation corrections) of the very brownish extracted colors, and computation of the color calibration transformation. This method led to an average CIE-94 color difference between the ground truth colors components of the color chart patches and the colors of the patches acquired under the microscope after calibration of 2.2 units, which is a fairly good accuracy compared to the results obtained in $[9,16]$, given the bad conditions of our microscope imaging system. The authors of these papers were not indeed faced with strong color distortion or surface inhomogeneities. The robustness of this method has been confirmed by applying it directly on microscope camera images of the patches and by comparing the colors extracted from these images and the ground truth colors of the patches. The obtained average CIE-94 (2.5 units) is only a little higher.

Another aspect studied here is the variation of color calibration accuracy with the choice of the reference color values, that is, the spectroscopic measurement configuration of the reflectance of the color chart patches as well as the illuminant chosen to compute the reference color components. As the chosen colorchart patches have a low specular component, the measurement geometry has low impact on the color calibration accuracy. We observed that the choice of the illuminant has higher influence and that the best calibration results are obtained for a blackbody illuminant of $1900 \mathrm{~K}$, which is the closest to the halogen illumination used for the microscope studied.

\section{Appendix A}

The color calibration of the camera is based on various steps. Figure 10 gives a synthetic view of the variation of the colors after each step. 
This work was supported by the LABEX MANUTECH-SISE (ANR-10-LABX-0075) of Université de Lyon, within the program "Investissements d'Avenir" (ANR-11-IDEX-0007) operated by the French National Research Agency (ANR).

\section{References}

1. R. W. G. Hunt, Measuring Colour (Fountain, 1998).

2. G. Sharma, Digital Color Imaging Handbook (CRC Press, 2002).

3. T. Johnson, "Methods for characterizing colour scanners and digital cameras," Displays 16, 183-191 (1996).

4. G. Wyszecki and W. S. Stiles, Color Science: Concepts and Methods, Quantitative Data and Formulae, 2nd ed. (Wiley, 1982).

5. G. Hong, M. R. Luo, and P. A. Rhodes, "A study of digital camera colorimetric characterization based on polynomial modeling," Color Res. Appl. 26, 76-84 (2001).

6. P.-C. Hung, "Colorimetric calibration for scanners and media," Proc. SPIE 1448, 164-174 (1991).

7. P. Colantoni, J. B. Thomas, and J. Y. Hardeberg, "High-end colorimetric display characterization using an adaptive training set," J. Soc. Inf. Disp. 19, 520-530 (2011).

8. R. Bala, G. Sharma, V. Monga, and J. P. V. de Capelle, "Twodimensional transforms for device color correction and calibration,” IEEE Trans. Image Process. 14, 1172-1186 (2005).

9. P. Jackman, D.-W. Sun, and G. ElMasry, "Robust colour calibration of an imaging system using a colour space transform and advanced regression modelling," Meat Sci. 91, 402-407 (2012).

10. H. R. Kang and P. G. Anderson, "Neural network applications to the color scanner and printer calibrations," J. Electron. Imaging 1, 125-135 (1992).

11. A. Artusi and A. Wilkie, "Novel color printer characterization model," J. Electron. Imaging 12, 448-458 (2003).

12. A. Neumann, A. Artusi, L. Neumann, G. Zotti, and W Purgathofer, "Accurate display gamma functions based on human observation," Color Res. Appl. 32, 310-319 (2007).

13. N. A. Valous, F. Mendoza, D.-W. Sun, and P. Allen, "Colour calibration of a laboratory computer vision system for quality evaluation of pre-sliced hams," Meat science 81, 132-141 (2009).

14. C. Grana, G. Pellacani, and S. Seidenari, "Practical color calibration for dermoscopy, applied to a digital epiluminescence microscope," Skin Res. Technol. 11, 242-247 (2005).

15. Y. V. Haeghen, J. M. A. D. Naeyaert, I. Lemahieu, and W. Philips, "An imaging system with calibrated color image acquisition for use in dermatology," IEEE Trans. Med. Imaging 19, 722-730 (2000).
16. J. Quintana, R. Garcia, and L. Neumann, "A novel method for color correction in epiluminescence microscopy," Comput. Med. Imaging Graph 35, 646-652 (2011).

17. W. Li, M. Soto-Thompson, and U. Gustafsson, "A new image calibration system in digital colposcopy," Opt. Express 14, 12887-12901 (2006).

18. A. Wee, D. Lindsey, S. Kuo, and W. Johnston, "Color accuracy of commercial digital cameras for use in dentistry," Dent. Mater. 22, 553-559 (2006).

19. J. Marguier, N. Bhatti, H. Baker, and S. Süsstrunk, "A home décor expert in your camera," in Proceedings of the IS\&T/SID 17th Color Imaging Conference (Society for Imaging Science and Technology, 2009), pp. 85-90.

20. V. Cheung, S. Westland, D. Connah, and C. Ripamonti, "A comparative study of the characterisation of colour cameras by means of neural networks and polynomial transforms," Color. Technol. 120, 19-25 (2004).

21. CIE, "Industrial color difference evaluation," CIE Publication No. 116 (Central Bureau of the CIE, Vienna, 1995).

22. CIE, "Colorimetry-official recommendations of the international commission on illumination," CIE Publication No. 15.2 (Central Bureau of the CIE, Vienna, 1986).

23. S. Stamm, "An investigation of color tolerance," in TAGA Proceedings, (Technical Association of the Graphic Arts, 1981), pp. $156-173$.

24. C. J. Dalton, "The measurement of the colorimetric fidelity of television cameras," J. Inst. Electron. Radio Eng. UK 58, 181-186 (1988).

25. K. Xiao, F. Zardawi, and J. M. Yates, "Colour management system for displaying microscope images," Displays 33, 214-220 (2012).

26. M. R. Luo, G. Cui, and B. Rigg, "The development of the CIE 2000 colour-difference formula: CIEDE2000," Color Res. Appl. 26, 340-350 (2001).

27. N. Destouches, N. Crespo-Monteiro, T. Epicier, Y. Lefkir, F. Vocanson, S. Reynaud, R. Charrière, and M. Hébert, "Permanent dichroic coloring of surfaces by laser-induced formation of chain-like self-organized silver nanoparticles within crystalline titania films," Proc. SPIE 8609, 860905 (2013).

28. CIE, "Colorimetry," CIE Publication 15, 3rd ed. (Central Bureau of the CIE, Vienna, 2004).

29. CIE, "International Lighting Vocabulary," CIE Technical Report, 4th ed. (1987).

30. P. Danny, "RGB coordinates of the MacBeth ColorChecker," (2006).

31. J. Lagarias, J. Reeds, M. Wright, and P. Wright, "Convergence properties of the Nelder-Mead simplex method in low Dimensions," SIAM J. Optim. 9, 112-147 (1998). 